\title{
Machine State Identification based on Information Fusion
}

\author{
Sun Zhiyuan ${ }^{\text {a }}$, Zheng Jian, Xiong Chao, Yin Junhui \\ Ordnance Engineering College, Shijiazhuang 050003, China; \\ asunzhiyuandII@163.com
}

Keywords: DS evidence theory; SVDD; BP neural network; Machine state identification

\begin{abstract}
Aiming at the uncertainty of the results obtained by using single sensor and single algorithm in the process of machine state identification, the DS evidence theory is introduced. Firstly, the basic probability assignment (BPA) is constructed according to the classification results of the support vector data description (SVDD) and BP neural network. Then the BPA of the two algorithms are fused according to the evidence rule, and the output result of the single sensor is obtained. Finally, the decision conclusion is obtained according to the fusion of multi sensor classification results. It can be seen from the bearing experiment that the uncertainty of decision is greatly reduced after information fusion, which can make full use of the complementary information of each signal sensor to improve the accuracy and credibility of the decision.
\end{abstract}

\section{Introduction}

Installing sensors in some key parts of the equipment for data acquisition and analysis is an effective method to grasp the performance and find out potential faults timely in the running of mechanical equipment. The data collected by each sensor directly reflect the different running states of the equipment. The running state of the equipment can be identified by using various algorithms such as neural networks and support vector machine (SVM). But due to the reliability of the sensor and the accuracy of the algorithm, the results obtained from a single information source are often uncertain, and it is easy to make wrong decision.

As a kind of important uncertain information processing method, the evidence theory can effectively fuse the information of different sensors and use the combination rule to dig out the components, and get the reasonable conclusion ${ }^{[1]}$. In this paper, the support vector data description (SVDD) algorithm and BP neural network are used to analysis the vibration signal of the bearing collected by the multi sensor, getting the evidence body. The evidence bodies are integrated effectively using Dempster's rule to achieve the effective identification of different states. The results show that the method can make full use of the information of the sensors and the algorithm, and greatly improve the accuracy of the results.

\section{Multiple states recognition based on SVDD}

SVDD is a commonly used method of single class based on support vector machine. The objective of SVDD is to find the best data description of target data in one-class classification. Assume a data set $\left\{x_{1}, x_{2} \ldots x_{\mathrm{n}}\right\}$ where $n$ is the number of target data. The goal of SVDD is to determine the data description as the smallest sphere containing all possible target data in feature space. The objective function of SVDD is as follow:

$$
\left\{\begin{array}{l}
\min f\left(a, R, \xi_{i}\right)=R^{2}+C \sum_{i} \xi_{i} \\
\text { s.t. }\left(\varphi\left(x_{i}\right)-a\right)\left(\varphi\left(x_{i}\right)-a\right)^{T} \leq R^{2}+\xi_{i} \quad \xi_{i} \geq 0
\end{array}\right.
$$

The variable $\xi_{i}$ is used to incorporate the effect of data not included in the spherical description. $R$ is the radius of the sphere used in SVDD

Detailed solution process please refers to the literature[2]. By solving a quadratic programming problem, the following conclusions can be obtained: 


$$
\begin{aligned}
& R=K\left(x_{k} \cdot x_{k}\right)-2 \sum_{i=1}^{n} \alpha_{i} K\left(x_{i} \cdot x_{k}\right)+\sum_{i, j=1}^{n} \alpha_{i} \alpha_{j} K\left(x_{i} \cdot x_{j}\right) \\
& d=K(z \cdot z)-2 \sum_{i=1}^{n} \alpha_{i} K\left(x_{i} \cdot z\right)+\sum_{i, j=1}^{n} \alpha_{i} \alpha_{j}\left(x_{i} \cdot x_{j}\right)
\end{aligned}
$$

$K(x, y)$ is the kernel function. $d$ is the distance of a sample to the sphere center.

Because the different sphere is obtained by different one-class sample set, there is often a big difference between the radiuses of the spheres. And the overlap between the spheres likely leads to a wrong judgment. The relative distance $H_{i}=d_{\mathrm{i}} / R$ is proposed to decide which sphere the test sample belongs to. $f_{i}$ is membership degree of the test sample $x_{i}$ and the sphere:

$$
f_{i}= \begin{cases}1 & h_{i} \leq a \\ e^{-\frac{\left(h_{i}-a\right)^{2}}{\sigma^{2}}} & h_{i}>a\end{cases}
$$

\section{BP neural network}

BP neural network is a multilayer feed-forward network based on the error response propagation algorithm. With a layer structure including input layer, hidden layer and output layer, BP neural network has the characteristics of distributed parallel processing, adaptive learning, nonlinear mapping and strong robustness, and is widely used in the field of intelligent control, pattern recognition and fault diagnosis ${ }^{[3]}$.

When the BP neural network is used as classifier, the number of output nodes is equal to the number of states. When training, the output node of the training sample is 1 , and the other output nodes is 0 . When the test sample is input into the neural network, the output is the membership degrees of the test sample to all kinds of states ${ }^{[4]}$.

\section{D-S evidence theory}

Dempster-Shafer theory dealing with uncertainty is an important method. D-S evidence theory can be considered to be a general extension of Bayesian theory and can robustly deal with incomplete data. Rather than computing probabilities of propositions, it computes probabilities that evidence supports the propositions and offers an alternative approach to dealing with uncertainty reasoning based on incomplete information ${ }^{[5]}$. The main process of the evidence theory is as follows:

\section{Basic probability distribution function of the evidence body}

Let $\Theta$ be a finite nonempty set of mutually exclusive alternative, and be called the frame of discernment. The frame of discernment contains every possible hypothesis. In mechanical equipment state identification, $\Theta$ is the set of condition of the equipment, $\Theta=$ \{normal $F_{1}$, fault $F_{2} \ldots$ fault $F_{n}$ \}.

Basic Probability Assignment (BPA) is a function, $m: 2^{\theta} \rightarrow[0,1]$, such that $m(\varnothing)=0$ where $\varnothing$ denotes an empty set, and $\sum_{A \subseteq \Theta} m(A)=1 . m(A)$ reflects the degree of the trust in the $A$ itself.

Considering the membership in SVDD and BP neural network,

$$
\left\{\begin{array}{l}
m_{k}\left(F_{i}\right)=\frac{h_{i} \beta_{k}}{\sum_{i=1}^{n} h_{i}} \\
m_{k}(\Theta)=1-\beta_{k}
\end{array}\right.
$$

$\beta_{k}$ is the reliability of the Kth evidence body, and is under the influence of installation of sensors, the accuracy of the algorithm and other factors. $m_{k}\left(F_{i}\right)$ is the BPA of the Kth evidence body. $m_{k}(\Theta)$ is the BPA of uncertainty.

\section{Evidence fusion}

In the same framework, different and independent evidences can be got according to the sources of sensors and Algorithm. Multiple evidences can be fused using Dempster's combination rule, shown in $\mathrm{Eq(6)}$, which is also called the orthogonal sum of evidences. 


$$
\left[m_{1}+m_{2}\right](A)=\frac{\sum_{A_{i} \cap B_{j}=A} m_{1}\left(A_{i}\right) m_{2}\left(B_{j}\right)}{1-\sum_{A_{i} \cap B_{j}=\varnothing} m_{1}\left(A_{i}\right) m_{2}\left(B_{j}\right)}
$$

\section{Decision rules}

The proposition is identified based on the results of the fusion. This paper chooses the decision-making method based on the BPA. The rules are as follows:

(1)The judgment proposition should have the maximum probability value

(2)The difference between the probability of the judgment proposition and the probability of other propositions should be greater than $\varepsilon_{1}$

(3)The probability of uncertainty should be less than $\varepsilon_{2}$.

(4) The probability of the judgment proposition should be greater than the probability of uncertainty.

\section{Experimental analysis}

The experimental data of this paper come from Case Western Reserve University. The states of the bearing equipment include the normal working state, the outer ring fault and the inner race fault. The two acceleration sensors are deployed on the device driver and the fan end by using the magnetic base to collect the vibration data in different state of equipment. The sampling frequency is $12 \mathrm{kHz}$ and the bearing speed is $1797 \mathrm{rpm}$. In each working state of the bearing, the vibration signal is collected by the two sensors and the signal is divided into 50 groups. In order to extract the feature parameters, the six characteristic parameters of the vibration signal are chosen as the input of SVDD and BP neural network, including mean, variance, kurtosis, skewness, root and form factor.

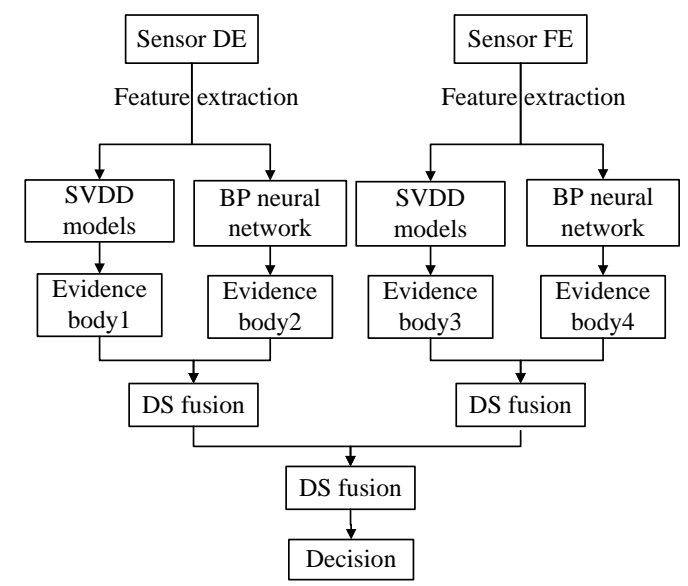

Figure 1 Multi sensor fusion model

Multi sensor fusion model for bearing state detection is shown in Figure 1. Taking any one sensor as an example, the first 25 groups of data are used as the training sample to establish the neural network and the 3 spheres for working states identification. The remaining 25 sets of data are used as the input of neural network and SVDD classification model. The membership degree of the test sample is obtained by calculation, and the BPA which constitutes the evidence bodies of information fusion is obtained. Some results which are the output of neural network and SVDD are shown in the table 1 and table 2. The fusion result of the sensor is obtained by the evidence bodies from the two kinds of algorithms. And the results are shown in table 3. Finally, the decision is made based on the multi sensor fusion results which are shown in table 4.

It can be seen in table 1 2 that using single sensor and single algorithm to identify the state can't have a higher accuracy and even sometimes get a wrong judgment. The sensor installation and the choice of identification algorithm has a great influence on the accuracy of the identification. Sometimes the degree of membership of the proposition is very close, resulting in the difficulty to make a correct judgment. Therefore, in the actual operation process, a single information source has a 
strong uncertainty. It can be seen that after the information fusion, the uncertainty of decision making is greatly reduced, which can make full use of the complementary information of each signal source to improve the accuracy and credibility of the decision.

Table 1 output indentification result of sensor DE

\begin{tabular}{|c|c|c|c|c|c|c|}
\hline \multicolumn{3}{|c|}{ F1: normal; } & \multicolumn{2}{c|}{ F2: Inner race fault } & \multicolumn{3}{c|}{ F3: Outer race fault } \\
\hline \multirow{2}{*}{$\begin{array}{c}\text { Test sample } \\
\text { number }\end{array}$} & output membership of SVDD & \multicolumn{2}{c|}{ output membership of BP NN } \\
\cline { 2 - 7 } & F1 & F2 & F3 & F1 & F2 & F3 \\
\hline 1 & 0.679 & 0.321 & 0 & 0.881 & 0.116 & 0.003 \\
\hline 2 & 0.35 & 0.65 & 0 & 0.05 & 0.892 & 0.058 \\
\hline 3 & 0 & 0 & 1 & 0.006 & 0.15 & 0.844 \\
\hline
\end{tabular}

Table 2 output indentification result of sensor FE

\begin{tabular}{|c|c|c|c|c|c|c|}
\hline \multicolumn{3}{|c|}{ F1: normal; } & \multicolumn{2}{c|}{ F2: Inner race fault } & \multicolumn{3}{c|}{ F3: Outer race fault } \\
\hline \multirow{2}{*}{$\begin{array}{c}\text { Test sample } \\
\text { number }\end{array}$} & \multicolumn{2}{|c|}{ output membership of SVDD } & \multicolumn{3}{c|}{ output membership of BP NN } \\
\cline { 2 - 7 } & F1 & F2 & F3 & F1 & F2 & F3 \\
\hline 1 & 0.436 & 0.254 & 0.31 & 0.929 & 0.064 & 0.007 \\
\hline 2 & 0.195 & 0.403 & 0.402 & 0 & 0.772 & 0.228 \\
\hline 3 & 0.248 & 0.376 & 0.376 & 0 & 0.158 & 0.842 \\
\hline
\end{tabular}

Table 3 fusion result of different algorthms

\begin{tabular}{|c|c|c|c|c|c|c|c|c|}
\hline \multirow{2}{*}{$\begin{array}{c}\text { Test sample } \\
\text { number }\end{array}$} & \multicolumn{4}{|c|}{ sensor DE fusion result } & \multicolumn{4}{c|}{ sensor FE fusion result of } \\
\cline { 2 - 9 } & F1 & F2 & F3 & Uncertainty & F1 & F2 & F3 & Uncertainty \\
\hline 1 & 0.866 & 0.113 & 0 & 0.021 & 0.799 & 0.086 & 0.066 & 0.049 \\
\hline 2 & 0.094 & 0.877 & 0.007 & 0.022 & 0.039 & 0.658 & 0.252 & 0.051 \\
\hline 3 & 0.001 & 0.014 & 0.968 & 0.017 & 0.052 & 0.195 & 0.702 & 0.052 \\
\hline
\end{tabular}

Table 4 fusion result of different sensors

\begin{tabular}{|c|c|c|c|c|c|}
\hline \multirow{2}{*}{$\begin{array}{c}\text { Test sample } \\
\text { number }\end{array}$} & \multicolumn{4}{|c|}{ Fusion result of sensors } & Decision \\
\cline { 2 - 5 } & F1 & F2 & F3 & Uncertainty & result \\
\hline 1 & 0.975 & 0.022 & 0.002 & 0.001 & F1 \\
\hline 2 & 0.014 & 0.973 & 0.012 & 0.002 & F2 \\
\hline 3 & 0.001 & 0.009 & 0.988 & 0.001 & F3 \\
\hline
\end{tabular}

\section{Conclusion}

The identification obtained by using single algorithm and single sensor sometimes results in the uncertainty and low credibility. By combining SVDD algorithm, BP neural network with DS evidence theory, different analysis methods used to deal with the signal from multiple sensors can make full use of the complementary information and improve the efficiency and accuracy. This method has high generality and practical value.

\section{References}

[1] Xianfeng Fan, Ming J. Zuo. Fault diagnosis of machines based on D-S evidence theory [J]. Pattern Recognition Letters, 2006, (27):366-376.

[2] Tax D M J. Duin R P W. Support vector domain description [J]. Pattern Recognition Letters,1999,20(11-13):1191-1199.

[3] Luwan Chen, Weihong Wang, Wenjun Zhang. Risk evaluation of regional collapses geological hazard based on D-S evidence theory[J]. Energy Procidia, 2012,(16):371-376.

[4] XU Weixiao, TAN Jiwen, WEN Yan. Study on fault diagnosis methods of rolling bearing based on BP neural network and D-S evidence theory [J]. Machine Tool\&Hydraulics, 2014, 42(23):188-191.

[5] Zhang Lingling, Liao Hongyun, Jia Jide, et al A research on engine fault diagnosis based on SVDD and D-S evidence theory[J]. Automotive Engineering, 2013, (35):23-26. 